\title{
The Necessity of Autonomous Evaluation of Parametric Modeling and Draft- ing Instruction
}

\section{Dr. Jeff P. Morris, Rensselaer Polytechnic Institute}

Jeff Morris is the technical manager of CAD/CAM/CAE for the School of Engineering at Rensselaer Polytechnic Institute, managing the computer-aided design/manufacturing/engineering training and curriculum for the school. Jeff received his B.S. in Mechanical Engineering from Rensselaer in 2002 and worked in the chemical-mechanical polishing industry for Dow (formerly Rohm \& Haas) for two years before beginning graduate studies. He completed a Master of Science in Mechanical Engineering in 2005 from Rensselaer, focusing on product assembly design. He completed his graduate work in 2011 with a doctorate in Engineering Science from Rensselaer, developing sustainability metrics for product assemblies. He continues research in the fields of sustainability and computer-aided-engineering curricula. 


\title{
The Necessity of Autonomous Evaluation of Parametric Modeling and Drafting Instruction
}

\begin{abstract}
Higher-educational STEM-focused institutions are finding it necessary to evaluate modeling skills with CAD software in a quicker and more consistent manner. This paper describes the history, process, and improvement opportunities in the grading of student-submitted CAD files for a large introductory CAD course (>250 students). During a first-year, 14-week collegiate CAD course, hundreds of students create and submit thousands of CAD files for evaluation. For a large course, current evaluation of student assignment quality requires the employment ratio of one teaching assistant to 10 students, whose dual role is to offer assistance with questions and grade assignments. Currently, course assignments are graded manually through a five-point rubric within the weekly course meeting time. Manual grading begets the following disadvantages: at least a third of the class time is spent purely evaluating assignments (translating to less time spent offering assistance), teaching assistants will have various levels of experience with $\mathrm{CAD}$, grading inconsistencies may exist between teaching assistants (even with following a rubric), students may leave class without being graded or have their grades omitted through clerical errors, and complex models require too much time to properly catch potential modeling errors. To address these issues, a novel program has been written that interfaces with a CAD software to grade each CAD assignment within a fraction of a second. The program seeks to interrogate common modeling and geometric errors that students encounter when learning 2D and 3D solid modeling practices, and deduct for these errors independently (i.e. not merely a comparison of "volumes"). The program extracts relevant file properties to a spreadsheet, compares the set of submissions against either a "master" file, or a set of standards controlled by the grader, and returns a grade for the assignment. Further, the program is highly customizable, and can be tailored to different modeling strategies of the course. An entire class of students (approx. 30 - 45) can be graded in less than a minute. A submission procedure to a course-based server for collection and feedback presentation to students will also be discussed. As engineering instructors, it is a necessary duty to ensure that all students receive impartial and consistent evaluation of their submitted work. This method and program strives to that end, while lessening the staff resources required to evaluate student's submissions.
\end{abstract}

\section{Introduction}

More higher-educational STEM-focused institutions are finding it necessary to evaluate introductory two-dimensional and three-dimensional parametric modeling skills with CAD software in a quicker and more consistent manner. Each term, hundreds of students register for these courses, yet knowledgeable evaluators are sometimes limited to a handful, and in extreme cases, a single instructor. In courses where there are multiple evaluators (often departmentappointed teaching assistants with limited CAD knowledge themselves), the current protocol of 
in-person evaluation of student submissions requires relatively large amounts of time per student-model, and even specific grading rubrics are not complete enough to capture many student failures within each assignment.

There is a variety of pedagogical methods employed in these courses, and the creation of "correct" models and drafts can be have multiple correct procedures [1], [2]. Yet, there are fundamental concepts in visualization and definitions of views that should always be taught in the beginning of CAD instruction. For example, in learning about two-dimensional sketching, a student should understand how the application presents its 3-D space, origin, and coordinate systems.

Further, mobile computing platforms are dominating the higher learning institution market, at least in the U.S, with price-points decreasing and sufficient hardware becoming ubiquitous across the top vendors. This lends to the ability for each student to own and use their CAD hardware anywhere and at any time. Practice is not limited to the classroom. Neither is content delivery: with online instruction (both pre-recorded and real-time) now available, the two largest bottlenecks remaining in the learning process are live assistance and evaluation, which will be discussed in this paper.

Some students require in-person assistance. This is impossible with video, written, or other oneway instructional media. The students want to be re-assured that their geometric creation process (e.g. 'button-selecting', visual comprehension) will yield the correct results. Some examples of common issues raised by beginner students are below:

- “How can I tell if I'm in sketcher?"

- "Is this the TOP or the LEFT view?"

- "My resource bar disappeared"

- "I cannot find where the last constraint exists"

Even creating simple parametric sketches can be a divergent process for beginners, unfamiliar with the dozens of commands that are concurrently present on the user interface. In this case, limited live feedback should be used to complement the student's learning; however, the focus of this paper is the evaluation of student work.

There are several questions to answer in evaluating student work:

1. Result: How does the model/sheet (drafting) compare to the 'correct' model/sheet?

2. Process: Were the 'healthiest' methods used to create these models?

3. Time: Was the model completed 'on-time'?

4. Integrity: Was the file completed solely by the authorized student?

For any assignment, all or only some of the result and process checks may be employed. All of these checks can be performed at any time. Each of these topics will be discussed further in the remainder of this paper. It is imperative that any specific evaluation criteria be treated as independent as possible from any other criteria. For example, merely checking that a sketch is "Fully-Constrained" is not a complete evaluation for best modeling practices. A sketch could 
achieve the status of "Fully-Constrained" while still employing the following unhealthy practices:

1. Auto-dimensions

2. All curves are reference curves

3. Use of "fixed”, "fix all”, “constant length”, or "constant angle” constraints

4. Sketch is not closed

5. Too many dimensions or geometric constraints

Of the dozens of CAD software packages on the market today, some have employed add-ins or third-party applications in accomplishing automatic grading, quality, and integrity checks: GraderWorks/SolidWorks [3] and Expert Model Analysis or Precision LMS from PTC. Ault and Fraser [4] used the latter to pilot automated grading for consistency and speed improvements in the evaluation process.

Siemens NX uses the module named Check-Mate to author and perform various quality assurance tests of models and drawings. Some instructors have used the OpenAPI architecture to incorporate automated grading solutions using Siemens NX [5].

Kirstukas and Morris [6] reviewed several automatic evaluation systems for use in collegiate courses, including Guerci's early framework for automatic grading and feedback [7], and GraderWorks, which is SolidWorks dependent and unusable for this course.

\section{Current Course Format}

The ENGR-1200 Introductory Graphics \& Computer Aided Design Course is a one-credit introductory course that meets once per week, 14 weeks, for 110 minutes per meeting. All students are required to have laptops with working CAD software. The course hires enough staff to maintain a maximum 10:1 student-to-assistant ratio; a class section enrollment between 40 and 50 students will employ five (5) teaching assistants during class-time. The total typical semester enrollment is between 250 and 300 students that must be divided into 8 or 9 sections due to seating constraints of laptop-ready rooms. Each section will have between 20 and 50 students. Graduate student teaching assistants are assigned to the course by their department (e.g. Mechanical Engineering, Biomedical Engineering), and will rarely have sufficient CAD experience. Undergraduate teaching assistants are hired directly through the Course Coordinator and they must be former students of who have received an " $\mathrm{A}$ ” in the course. 


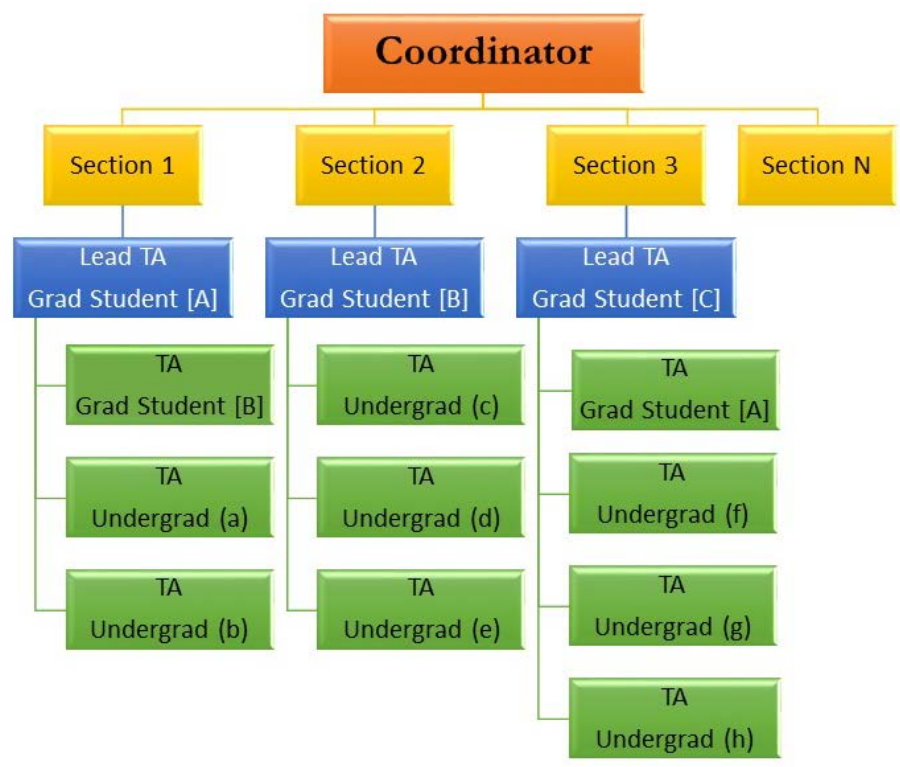

Figure 1: ENGR-1200 Leadership Hierarchy

At on the onset of the week, students are able to access all weekly lab assignments online and are encouraged to attempt each before attending class for that week. Once class begins, each student has the class period to receive assistance and have their work evaluated by a teaching assistant, but must 'submit' their final versions for grade by the end of class time. Submission is defined as:

1. Student raises their hand.

2. An available TA sits down alongside the student.

3. The TA commandeers the student's laptop for the purposes of interrogating their work to ensure the grading criterion has been met.

Most assignments are presented as standard drawings similar to Figure 2, with the shape, parameters, and orientation given to the student. The grading criteria always consist of five (5) objectives, which may change depending on assignment [1], [2]. For example, the "Track Plate" in Figure 2 serves to reinforce the topics of 3D orientation, extrusion symmetry, draft angles, and sketch constraints. This is a typical assignment in the third week. 

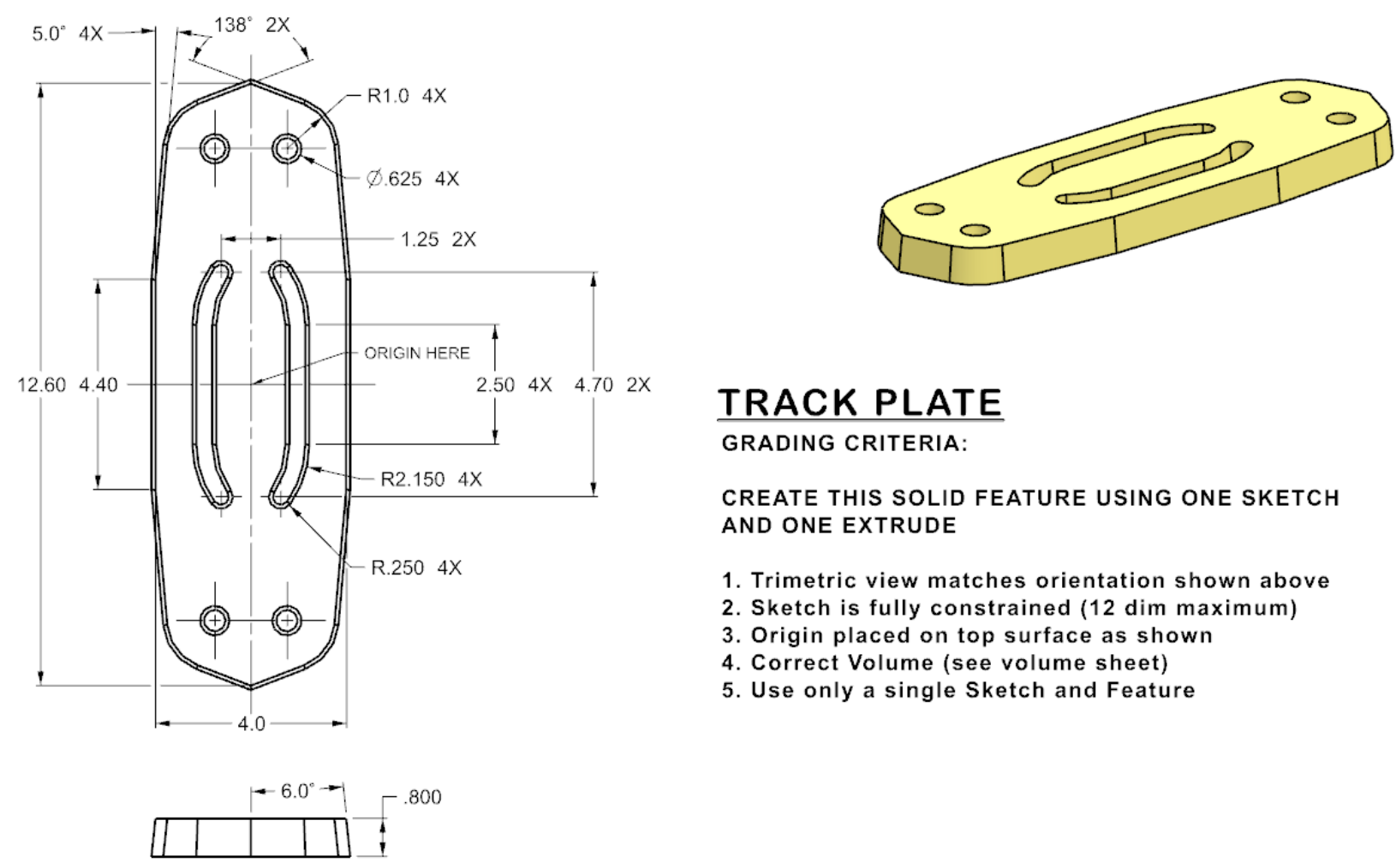

\section{TRACK PLATE}

GRADING CRITERIA:

CREATE THIS SOLID FEATURE USING ONE SKETCH AND ONE EXTRUDE

1. Trimetric view matches orientation shown above

2. Sketch is fully constrained ( $12 \mathrm{dim}$ maximum)

3. Origin placed on top surface as shown

4. Correct Volume (see volume sheet)

5. Use only a single Sketch and Feature

Figure 2: Example Sketch \& Extrude Assignment

The following in-classroom cases are witnessed:

- $\quad$ Student A believes he/she has met all five grading criteria prior to class start or during class time. An available evaluator evaluates the work and finds no mistakes. Student A may exit the class. This is the quickest case, and the evaluation process ranges from $30-$ 180 seconds per assignment.

- $\quad$ Student B believes he/she has met all five grading criteria prior to class start or during class time. An evaluator is not free, as all are currently offering assistance or evaluating other students. Student B must wait for an available evaluator. If an evaluator does not become available, the student may be forced to leave without getting credit for work completed. The longer a student takes to finish their assignments during class, the more likely this case is to occur.

- $\quad$ Student $\mathrm{C}$ believes he/she has met all five grading criteria prior to class start or during class time. A free evaluator begins an evaluation and flags a mistake(s). Student $\mathrm{C}$ may elect to fix them without assistance or ask the TA for assistance in fixing them. This process may take up to (10) minutes or longer. Student $C$ must fix these mistakes or be graded "as-is" if there is no class time remaining.

- $\quad$ Student D does not believe he/she has met all five grading criteria prior to class start. While he/she has attempted the assignments prior to class, he/she still requires assistance to complete the assignment(s). If no TA is available, the student most likely asks their peers and will continue to work and/or wait. This is the most common case. 
- $\quad$ Student E does not believe he/she has met all five grading criteria prior to class start. $\mathrm{He} /$ she has not viewed the lectures nor attempted the assignments prior to class. Course staff will explicitly discourage students from pursuing this track. The timeline of the course does not permit one-on-one in-class assistance for the duration an entire assignment. Evaluators will be reluctant to guide this student and instead, direct them to office hours.

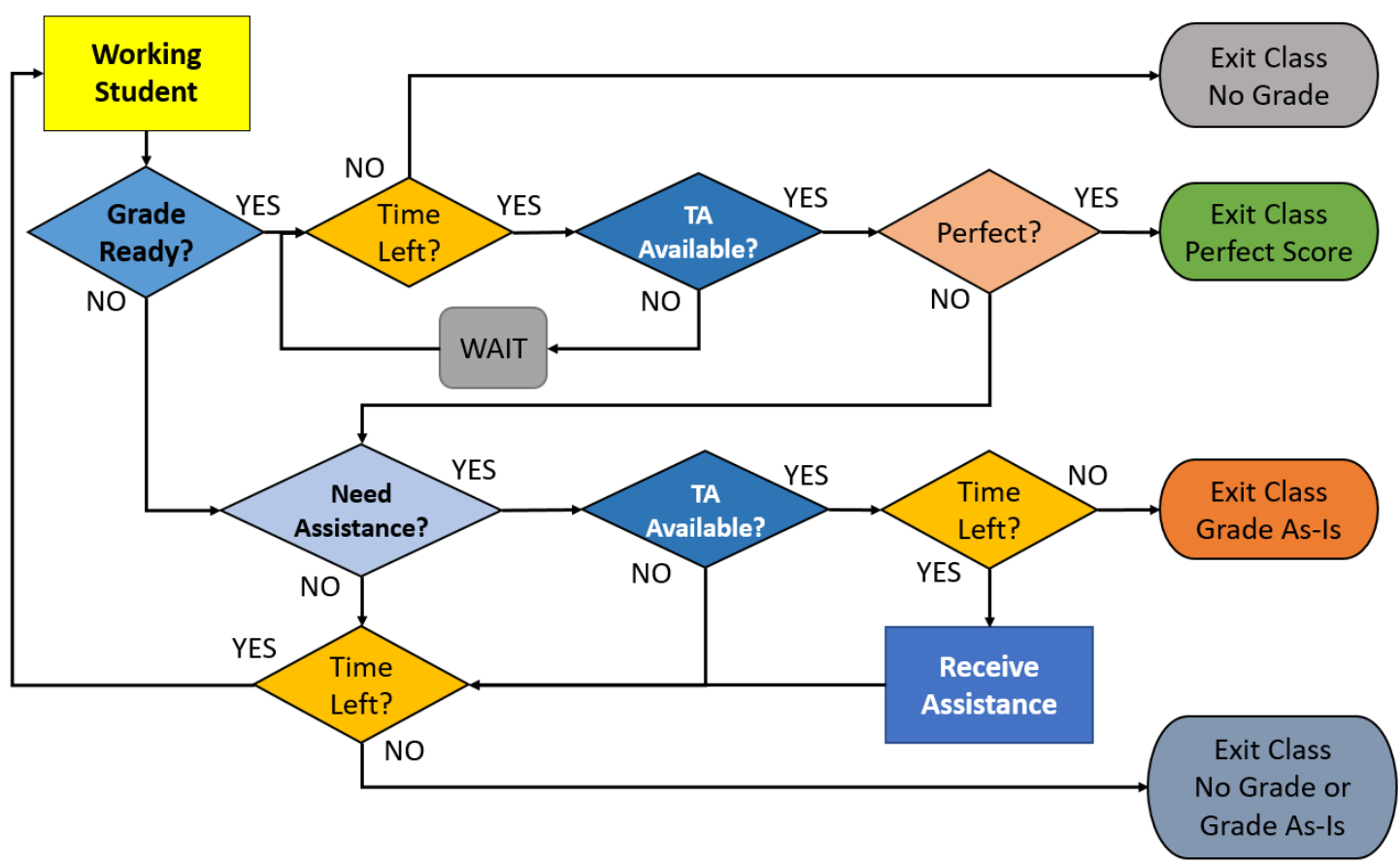

Figure 3: Current Student - Evaluation Workflow for All Possible Cases

Figure 3 captures the current possible states of a student as he/she attempts to complete an assignment and get evaluated within class. If all evaluators are busy assisting or grading other students, students requiring an evaluator for grading or assistance must wait. If there are many students requiring in-person assistance simultaneously, a queue of 'grade-ready' or 'assistanceseeking' students can form, resulting in the following:

a) The evaluation of students is 'rushed' and may not be accurate, leading to grading inconsistencies within the sections and across the course.

b) The assistance given to students in need is rushed, terse, and may not be sufficient to answer student's questions.

c) Due to time expiring, students may leave the class with no grades, through no fault of their own. This places an enormous challenge for the coordinator to interrogate files for their creation timestamps and re-grade at the conclusion of the course.

At least 30 percent of the class-time is spent evaluating student work. This time should be reduced so that students can have their submissions checked quickly and receive in-person assistance for learning rather than solely for evaluation. 
Moreover, there are many instances of teaching assistants incorrectly giving perfect scores to imperfect submissions due to one of the following:

a) lack of time (TA is rushed and overlooks checkpoints of failure)

b) lack of knowledge (TA does not know how to examine the checkpoints of failure)

c) lack of a complete grading rubric to specify necessary checkpoints of failure

\section{Automated workflows}

The following submission workflows are ranked from most to least ideal:

Workflow \#1: Remote Evaluation with Automatic Feedback

Figure 4 displays a workflow where students will upload their submission file(s) to a web server at their convenience, prior to its due date. The program will be tied into the registration system and generate an automatic e-mail to the student with their grade. If the result is acceptable to the student, nothing further is required. If the assignment is incorrect and if time remains, the student can modify and/or receive live assistance if available, and re-submit as many times as necessary until time expires. If class time expires, the last valid submission is taken as the assignment grade.

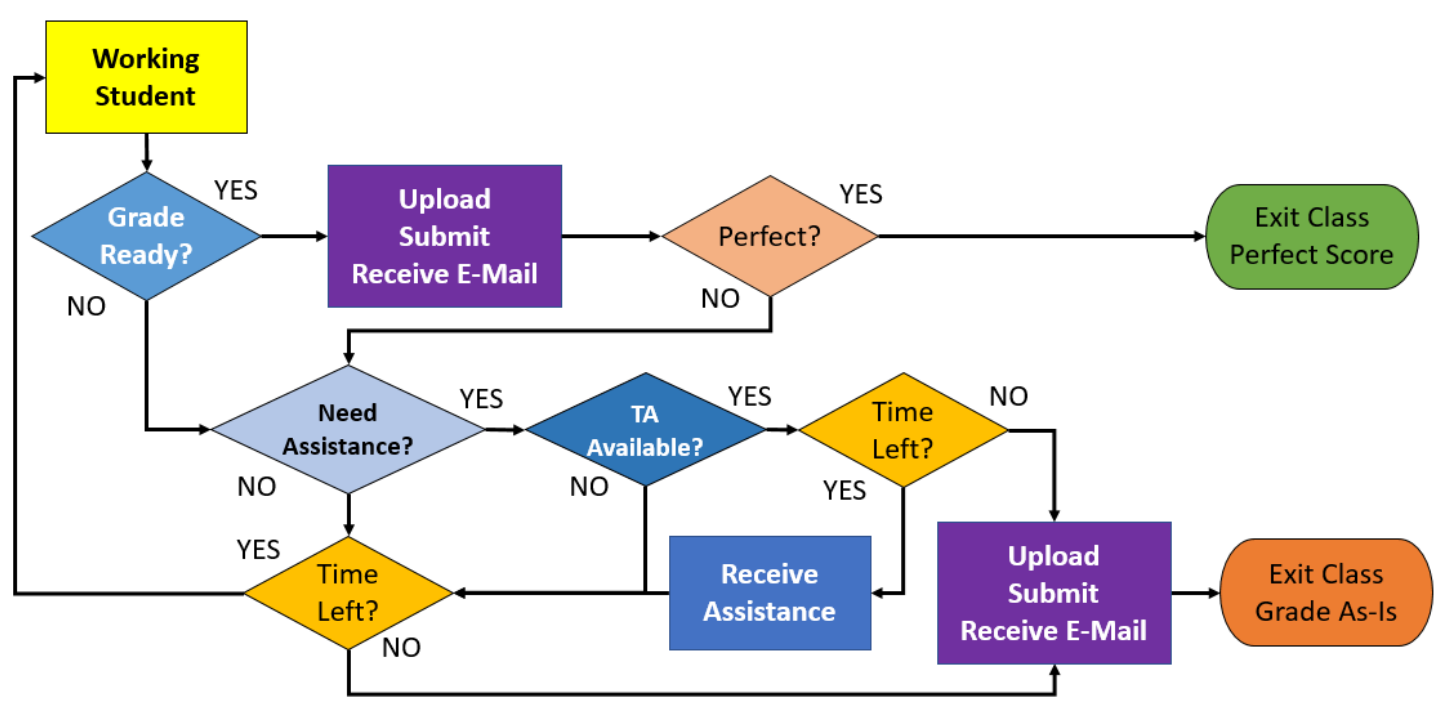

Figure 4: Ideal Online Evaluation Workflow with E-Mail Response

Workflow \#2: Client-based Evaluation with Automatic Feedback

In Figure 5, the CAD software includes an add-in application or is programmed to directly evaluate the geometry. The grading criteria file may be accessed remotely or downloaded to each student's workstation. A teaching assistant may oversee the use of the grading program to ensure validity and record the grade. 


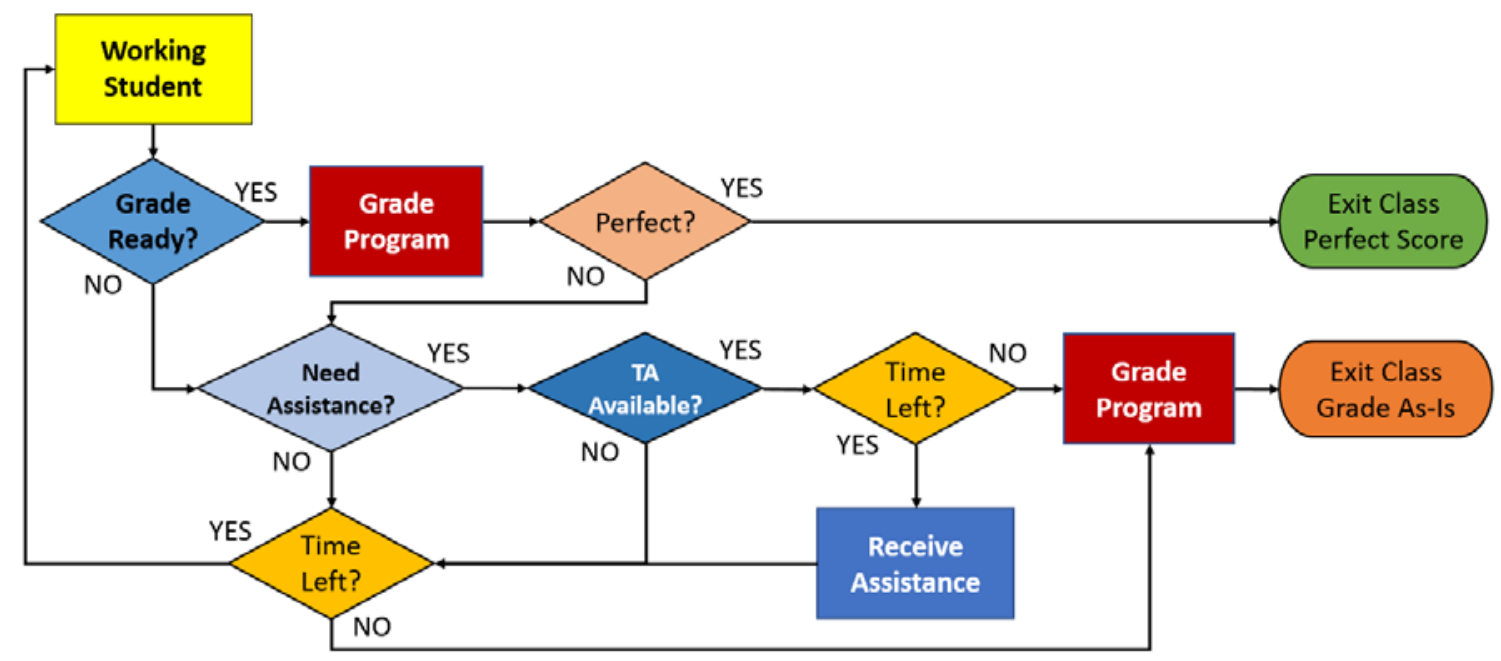

Figure 5: Client-based Evaluation Workflow

Workflow \#3: Remote Evaluation with Manual Feedback (as written for this paper)

As displayed in Figure 6, students will upload their submission file(s) to a directory at their convenience, prior to its due date. The evaluator will run the program. If the assignment is perfect, nothing further is required. If the assignment is incorrect, the student is made aware immediately via e-mail or in-class. If time remains, the student can modify and/or receive live assistance if available, and re-submit as many times as necessary until time expires. When time expires, the last valid submission is taken as the assignment grade.

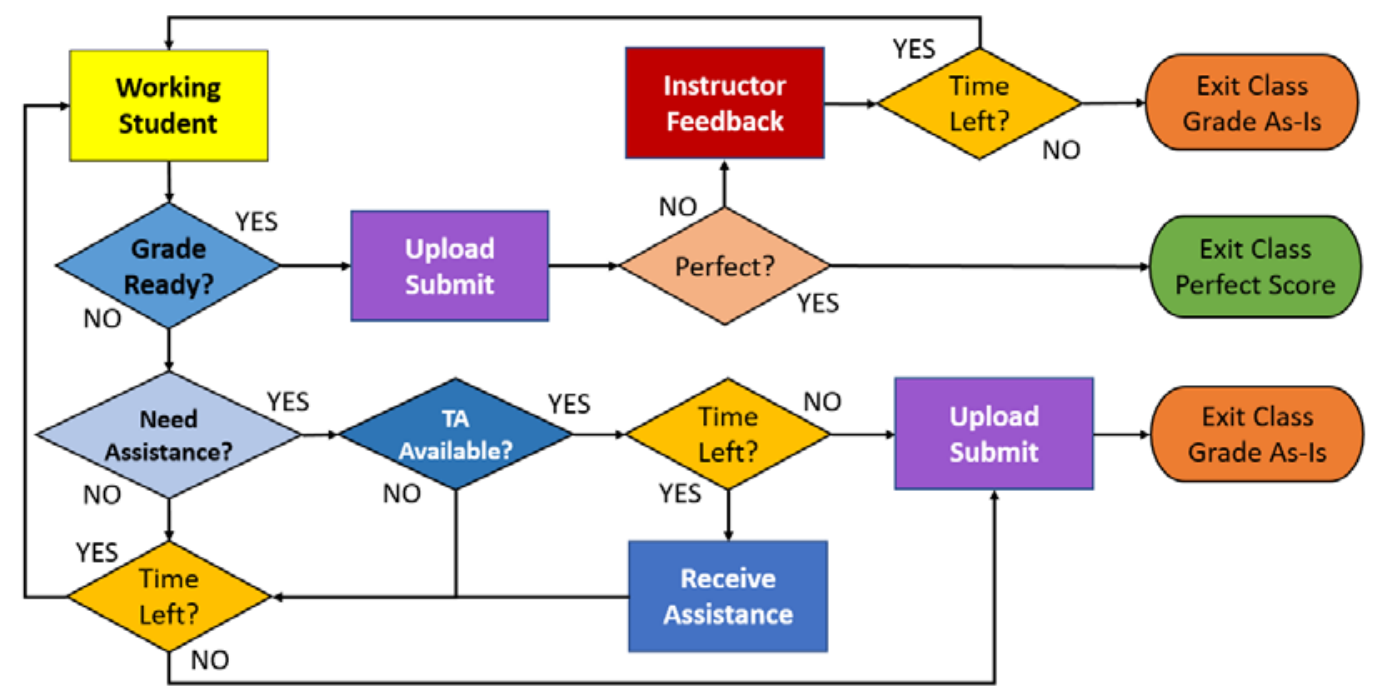

Figure 6: Remote Evaluation with Manual Feedback 
Workflow \#4: Evaluation with Score and No Feedback

Figure 7 depicts a scenario where students are given assistance at their request throughout the class time and are required to upload their final submissions by class expiration. Evaluators will grade the assignments later. There is no immediate feedback of student submissions scores. Students will undoubtedly request confirmation from evaluators that their submission is "ready." Evaluators will need to refrain from guaranteeing scores in-class, prior to grade.

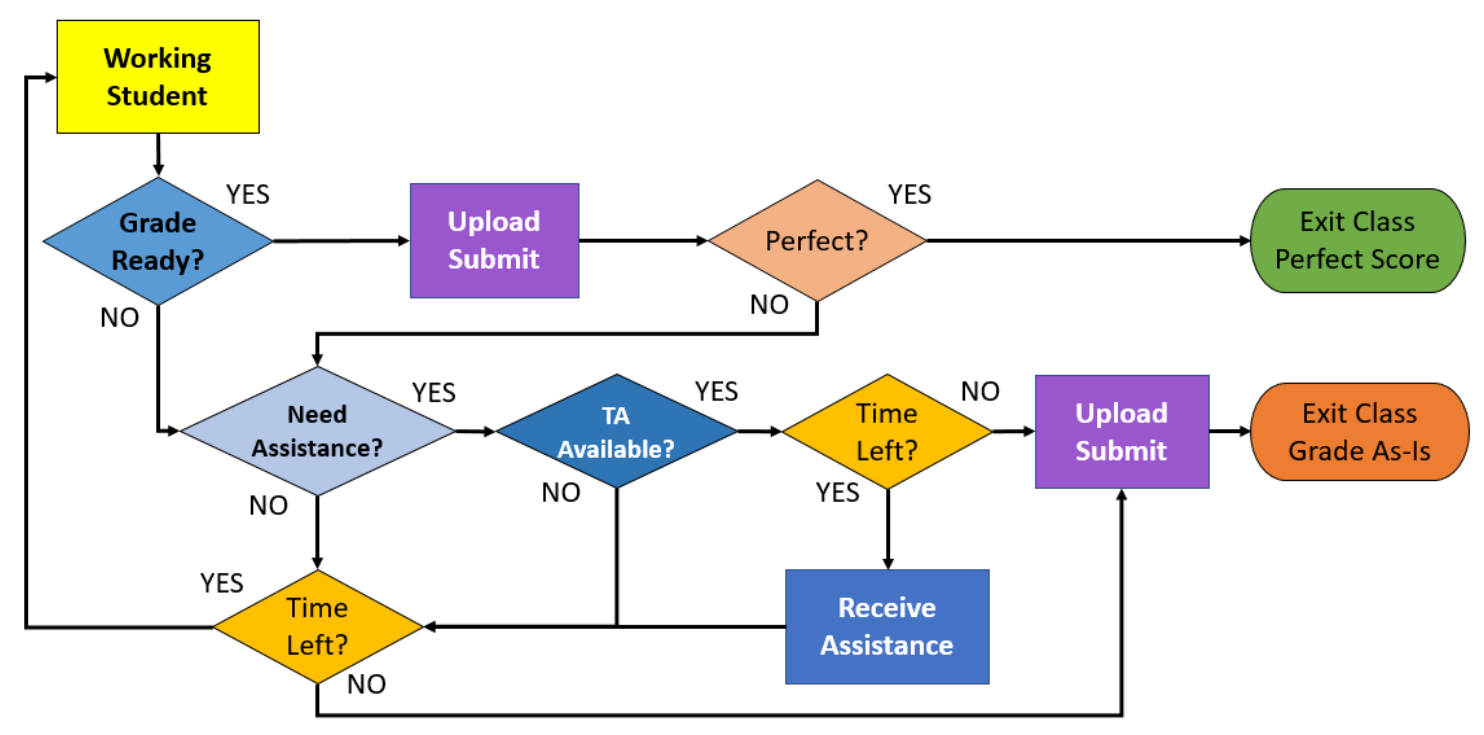

Figure 7: Upload with No Feedback

\section{Evaluation}

The following properties and checks are investigated for a complete score categorized as follows:

\section{Result}

The following checks could be employed to evaluate the result of models. Some checks can be compared to an instructor provided "Solution File".

\section{a) Solid Model vs. Solution File}

i) Unit Check (mm or inches)

(1) If $\mathrm{mm}$, then scale (penalize for $\mathrm{mm}$ )

ii) Body Orientation

(1) Moment of Inertias

iii) Placement

(1) Centroid Location relative to Absolute Coordinate System

iv) Shape

(1) Volume OR Moment of Inertia summation

(2) Surface Area 
(3) No. Edges

(4) No. Faces

v) Solid Body Count

(1) Zero Bodies

(2) Wrong Number of Bodies

vi) Model "Similarity" Percentage

(1) An aggregate of the shape scores

\section{b) Sketches vs. Solution File}

i) Dimension values

(1) Symmetric Values, Radii vs. Diameters, etc.

ii) Invalid Sketches (extra or open segments)

iii) Sketch Plane Orientation

iv) Sketch Placement

(1) Sketch Center of Gravity location relative to Absolute Coordinate System

\section{Process}

The following items should evaluating for 'healthiest practices'. For example, a student could use the 'fix' geometric constraint or have under-constrained sketches and still produce a perfect solid model that would satisfy all of the result checks. In introductory parametric modeling, this is not a preferred or taught method. The following checks are employed to evaluate the student process of construction:

a) Solid Model vs. Solution File

(1) Feature existence/count pre-set by instructor

(a) Examples: requirements to use circular pattern, hole, revolve, mirror, etc

(2) CSYS Count

(3) Datum Count

(4) Material Type

b) Sketches vs. Solution File

i) Count of unused sketches

ii) Count of empty sketches

iii) Count of sketches that are not fully constrained

(1) Not Fully Constrained = Under/Over/Not Solved/Auto-Dimensions

iv) Existence or non-existence of specific constraints pre-set by instructor

(1) Examples: no. of tangencies $=3$, no. of radial dimensions less than 2

(2) Fixed, Constant length, Constant angle

v) Minimum/maximum number of active dimensions

vi) Count and name of user-edited expressions

vii) Correct principal sketch plane 


\section{Time}

CAD instruction, along with most other computer-aided software learning is best delivered in the 'crawl-walk-run' approach. Offering simplistic geometry at the onset of the course and evaluating students on their abilities to grasp early 3-D visualization and 2-D sketch concepts is a necessary requirement before multiple-feature parts, drafting, and assembly work can begin. Hence, students must be evaluated and given feedback in sequential stages in order to highlight weaknesses and improve before attempting tasks that are more difficult. Student submission timestamps, therefore, must be compared with pre-set due dates to ensure students are maintaining chronologically consistent attempts at practice and assignment submission in accordance with the course calendar.

\section{Integrity}

While there are currently NO applications, scripts, software, or hardware applications that will $100 \%$ guarantee that a particular student solely created $100 \%$ of the digital file they submit, Morris details the automatic integrity checking process for submitted assignments as quick and comprehensive [8].

\section{Automatic Evaluation Software}

The author has written an application which interfaces with Siemens NX to evaluate numerous assignments in comparison to a solution file or other grading criteria. Currently, due to upload server infrastructure non-existence, files are evaluated at the end of the course after manual collection for beta-testing.

With the enhanced flexibility and speed of the automatic grading program, a more refined approach is possible. Figure 8 depicts the desired scoring page for the track-plate assignment. 


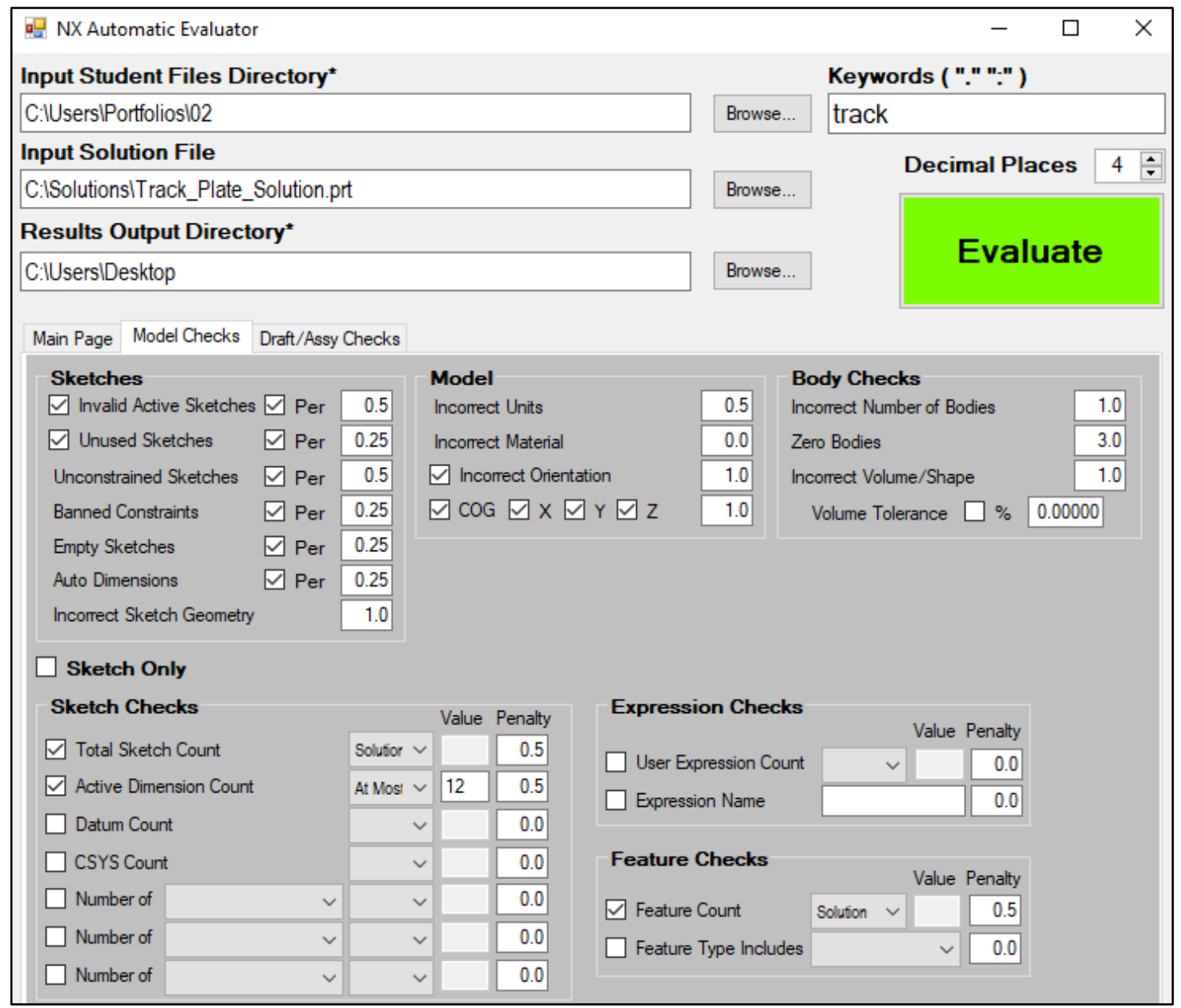

Figure 8: Auto-grader customization screen for the track-plate assignment

The automatic program can evaluate more checks per file than the teaching assistants are directed to or could comfortably perform in the allotted class time. For manual evaluation, typically only three - six checks per assignment are required [2]. Table 1 summarizes the checks for the track-plate assignment shown in Figure 2. 
Table 1: Evaluation Rubric Comparison for Single Extrude "Track-Plate”

\begin{tabular}{|c|c|c|c|}
\hline Check & Manual & Auto Old & Auto New \\
\hline Model Orientation & $\checkmark$ & $\checkmark$ & 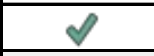 \\
\hline Under Constrained Sketch & $\vartheta$ & $\vartheta$ & $\checkmark$ \\
\hline Dimension Count & ] & $\vartheta$ & $\checkmark$ \\
\hline Origin Placement & 8 & $\checkmark$ & 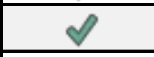 \\
\hline Volume & ] & $\checkmark$ & $\checkmark$ \\
\hline Sketch Count & $\vartheta$ & $\vartheta$ & $\vartheta$ \\
\hline Feature Count & $\vartheta$ & $\vartheta$ & $\vartheta$ \\
\hline Body Count & ] & 2 & 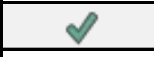 \\
\hline Auto-Dimensions & $x$ & $x$ & $\vartheta$ \\
\hline Unused Sketches & $x$ & $x$ & $\checkmark$ \\
\hline Empty Sketches & $\mathrm{x}$ & $x$ & $\checkmark$ \\
\hline Correct Units & $x$ & $x$ & $\vartheta$ \\
\hline Banned Constraints & x & $x$ & 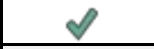 \\
\hline Invalid Sketches & $x$ & $x$ & $\checkmark$ \\
\hline Late/On-Time & ] & 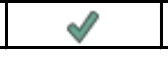 & $\checkmark$ \\
\hline \multicolumn{4}{|c|}{$\begin{array}{l}\text { Rapid Check (<5 s) } \\
\text { Not Easily Apparent }\end{array}$} \\
\hline
\end{tabular}

While it is possible that the items not evaluated could be done so manually (e.g. checking for "correct units", “empty sketches”, etc.), even experienced teaching assistants will miss several items during a session. Some checks require multiple clicks and mouse movements (e.g. Volume) and will slow the evaluation rate.

\begin{tabular}{|c|c|c|c|c|c|c|c|c|c|c|c|c|c|c|c|c|c|c|}
\hline SOL & SOL & SOL & SOL & & SOL & track_plate & & & IN. & 1 & 32.85 & 129.1093 & 98 & 36 & 0 & 0 & \begin{tabular}{l|l}
-0.408 \\
\end{tabular} & \\
\hline No. & TOTAL & SIM \% & SEM & SEC & ID & FILENAME & EXC & LATE & UNITS & NBOD & VOL & SA & EDGE & FACE & COG-X & COG-Y & COG-Z & \\
\hline 1 & 0.75 & 74.237 & FL18 & 4 & student 1 & track plate-1 & 0 & 1 & IN. & 1 & 28.83 & 121.178 & 98 & 36 & 0 & 0 & 0.3905 & \\
\hline 2 & 0 & 66.722 & FL18 & 4 & student 2 & track plate- 2 & 0 & 12 & IN. & 1 & 27.241 & 115.0945 & 98 & 36 & 0 & 0 & -0.4046 & \\
\hline 3 & 0 & 74.458 & FL18 & 4 & student 3 & track plate- 3 & 0 & & IN. & 1 & 28.88 & 121.3754 & 98 & 36 & 0.0012 & 0.0035 & 0.3905 & \\
\hline 4 & 3 & 98.036 & FL18 & 4 & student4 & track plate- 4 & 0 & & IN. & 1 & 32.77 & 129.2963 & 98 & 36 & 0 & 0 & -0.4081 & \\
\hline 5 & 0.001 & 100 & FL18 & 4 & student5 & track plate- 5 & 0 & 12 & IN. & 1 & 32.85 & 129.1093 & 98 & 36 & 0 & 0 & -0.408 & \\
\hline 6 & 3 & 73.182 & FL18 & 4 & student6 & track plate- 6 & 0 & & IN. & 1 & 28.317 & 119.7973 & 98 & 36 & 0 & 0 & 0.3903 & \\
\hline 7 & 1.5 & 87.321 & FL18 & 4 & \begin{tabular}{|l|} 
student 7 \\
\end{tabular} & track plate-7 & 0 & 1 & IN. & 1 & 34.977 & 133.0966 & 98 & 36 & 0 & 0 & \begin{tabular}{|l|}
-0.4073 \\
\end{tabular} & \\
\hline 8 & 3 & 22.736 & FL18 & 4 & student8 & track plate- 8 & 0 & & IN. & 1 & 87.419 & 693.0919 & 22 & 22 & 0 & -5.1648 & 0 & \\
\hline 9 & 4 & 100 & FL18 & 4 & student9 & track plate- 9 & 0 & & IN. & 1 & 32.85 & 129.1093 & 98 & 36 & 0 & 0 & -0.408 & \\
\hline 10 & 5 & 100 & FL18 & 4 & student10 & track plate- 10 & 0 & & IN. & 1 & 32.85 & 129.1093 & 98 & 36 & 0 & 0 & -0.408 & \\
\hline SOL & SOL & SOL & SOL & SOL & SOL & track_plate & 112.219 & 12.438 & 123.67 & 0.2829 & 1 & 0 & 0 & 0 & 0 & 12 & 0 & 1 \\
\hline No. & TOTAL & SIM \% & SEM & SEC & ID & FILENAME & MOI-X & MOI-Y & MOI-Z & DENS & STOT & SNC & SEMP & SNUS & AUTD & ACTD & FIX & $\mathbf{F C}$ \\
\hline 1 & 0.75 & 74.237 & FL18 & 4 & student1 & track plate-1 & 98.2318 & 10.251 & 107.61 & 0.2829 & 4 & 0 & 3 & 3 & 0 & 12 & 0 & 1 \\
\hline 2 & 0 & 66.722 & FL18 & 4 & student2 & track plate- 2 & 84.4282 & 8.7303 & 92.337 & 0.2829 & 1 & 0 & 0 & 0 & 0 & 15 & 0 & 2 \\
\hline 3 & 0 & 74.458 & FL18 & 4 & student 3 & track plate- 3 & 98.5058 & 10.266 & 107.9 & 0.2829 & 1 & 1 & 0 & 0 & 0 & 16 & 6 & 1 \\
\hline 4 & 3 & 98.036 & FL18 & 4 & student4 & track plate- 4 & 112.124 & 12.179 & 123.32 & 0.2829 & 1 & 0 & 0 & 0 & 0 & 11 & 0 & 1 \\
\hline 5 & 0.001 & 100 & FL18 & 4 & student5 & track plate- 5 & 112.219 & 12.438 & 123.67 & 0.2829 & 1 & 0 & 0 & 0 & 0 & 12 & 0 & 1 \\
\hline 6 & 3 & 73.182 & FL18 & 4 & student6 & track plate- 6 & 98.0405 & 10.187 & 107.37 & 0.2829 & 1 & 0 & 0 & 0 & 0 & 12 & 0 & 1 \\
\hline 7 & 1.5 & 87.321 & FL18 & 4 & student7 & track plate-7 & 113.621 & 12.987 & 125.55 & 0.2829 & 1 & 0 & 0 & 0 & 0 & 12 & 0 & 1 \\
\hline 8 & 3 & 22.736 & FL18 & 4 & student8 & track plate- 8 & 415.679 & 247.85 & 415.68 & 0.2829 & 1 & 0 & 0 & 0 & 0 & 10 & 0 & 1 \\
\hline 9 & 4 & 100 & FL18 & 4 & student9 & track plate- 9 & 112.219 & 12.438 & 123.67 & 0.2829 & 2 & 1 & 0 & 0 & 0 & 12 & 0 & 2 \\
\hline 10 & 5 & 100 & FL18 & 4 & student10 & track plate-10 & 112.219 & 12.438 & 123.67 & 0.2829 & 1 & 0 & 0 & 0 & 0 & 12 & 0 & 1 \\
\hline
\end{tabular}

Figure 9: Auto-grader output for ten student submissions of track-plate assignment

The column labeled "TOTAL" in Figure 9 above is the student score out of 5 points. Other highlighted items indicate deviations from the solution file (top row). The automatic program 
evaluates an entire section of students (30 file submissions) in approximately 30 seconds. It is customized to output a final part score similar to the existing manual rubric for comparison purposes. The program also yields a "Shape Similarity Percentage" to the solution file for reference purposes (currently this value does not affect the TOTAL score).

\section{Results}

The software was customized, as shown in Figure 10, to best match deductions on the assignment grading rubric in Figure 2.

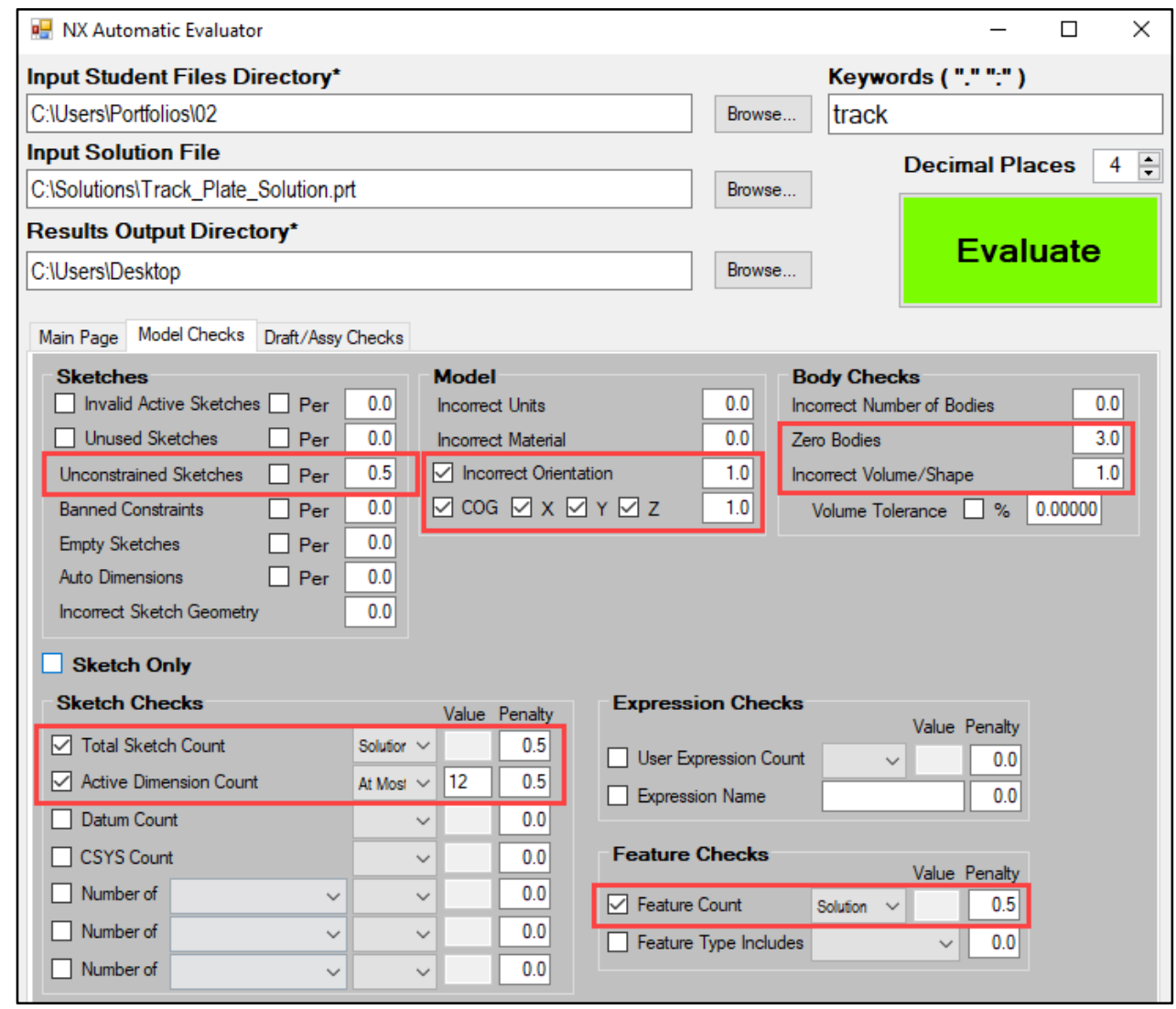

Figure 10: Auto-grader customization set to match current rubric

A comparison from the fall 2018 student submissions of the "track plate" assignment (see Figure 2) is displayed in Figure 11. Manual "TA Graded" scores in each section were compared against the automatic grading software using two different customizations: "AG Old” (Auto-Grader set to similar scoring of the rubric as shown to the students, see Figure 10) and "AG New" (AutoGrader configured for more checks and a finer resolution in grading, see Figure 8). 


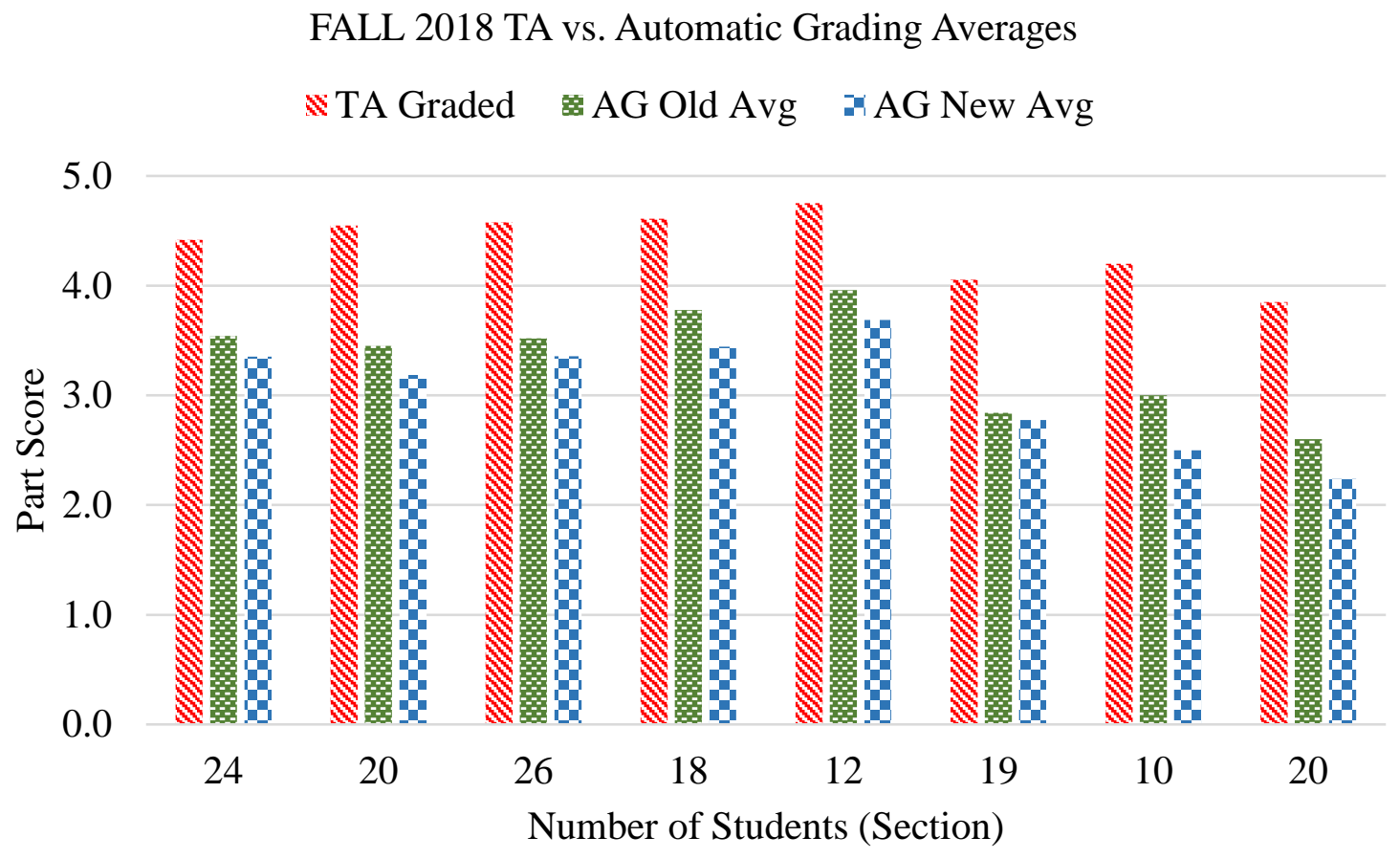

Figure 11: Comparison of Manual and Automatic Grading

The average difference between automatic and manual grading across sections and different teaching assistants is inflated between 17 to 27 percent. The preceding scores of manual TA graded files are captured across seven sections, comprised of seventeen different graders and 149 students. Students submitting late work $(\mathrm{n}=104)$ were not included in this comparison.

To further support the hypothesis of extensive manual grading errors, all non-late submissions for the "track-plate" assignment that were graded with a perfect score by TAs were filtered for any incorrect criteria items flagged by the automatic grader. Figure 12 shows a significant number of students were incorrectly graded for various criteria. For example, 15 students had an incorrect volume, 16 students had an under-constrained sketch, and yet these deficiencies were missed by teaching assistants. Only 40 out of 82 students that received perfect scores had no errors (i.e. were graded correctly) through manual grading. For the entire course, only 45 out of 149 students (30\%) were graded consistent with the given rubric. 
No. Students violating grading criteria, yet graded manually perfect $(5 / 5), n=82$

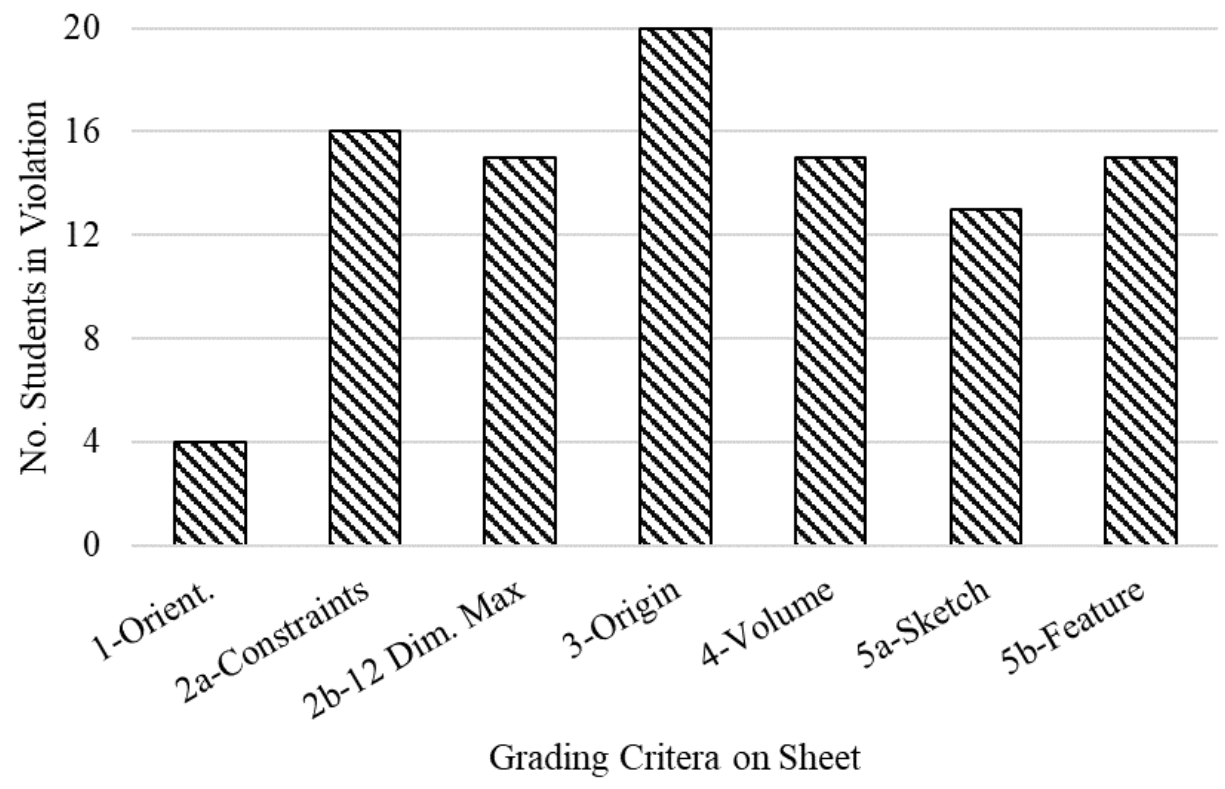

Figure 12: No. of Students Graded Perfect yet Incorrect by Manual Grading

\section{Conclusions}

A staggering $70 \%$ of student submissions for a class assignment were graded inconsistent with the assignment rubric. This data collected in fall 2018 suggested that teaching assistants, due to inexperience and sheer volume of student work to inspect, incur a 15 - 26 percent average grade inflation error with assignments. Due to these findings, an automatic software grader is necessary for large enrollments.

The grader may customize some or all of the Result and Process evaluations for each assignment. For example, in advanced multiple feature parts, the instructor may not check for correct sketch planes or a maximum/minimum number of dimensions. For simple 2D sketch assignments, performing volume and material checks are moot. The time and integrity checks should and could be run for every assignment submitted. The file integrity evaluation is included as an option in this application, and is performed at the conclusion of the course, comparing all submissions of all students against each other, even those in previous semesters.

Often, students waste class time waiting for a teaching assistant to become available to evaluate their work. Most beginning students judge their 'completeness' on the visual comparison between their sketch or model and the views or model given. It is common for a beginning student to believe they are finished prematurely; in their opinion, the model or sketch 'looks right' to the naked eye, yet it may have several mistakes. Rather than having them wait for an evaluator to become available, it would be beneficial for them to have a rapid and autonomous check to respond with their points of failure. Further, the automatic grading software has been 
shown to accurately and consistently flag a significant amount of modeling bad practices and deficiencies that teaching assistants will often miss.

\section{Future Work}

The author will begin investigation into automatic 2-D drafting comparisons and assembly model comparisons. The current version of NX does not have the capability to send the correct degreesof-freedom of assembly components to the API, which prevents a critical grading check to occur. The secure server infrastructure required for real-time upload of student submissions during the semester is currently under construction. Once completed, students will be asked to upload assignments upon completion, and the program can evaluate them in batch mode.

\section{References}

[1] T. J. Branoff, E. Wiebe and N. Hartman, "Integrating Constraint-Based CAD into an Introductory Engineering Graphics Course: Activities and Grading Strategies," in ASEE Annual Conference \& Exposition, Nashville, 2003.

[2] D. Baxter, "Evaluating Student Performance in a Freshman Graphics Course to Provide Early Interverntion for Students with Visualization and/or Design Intent Difficulties," in ASEE Annual Conference \& Exposition, Montreal, 2002.

[3] A. Garland, "GraderWorks," Garland Industries, LLC, [Online]. Available: https://garlandindustriesllc.com/index.php/pages/view/graderworks. [Accessed 14 August 2018].

[4] H. K. Ault and A. Fraser, "A Comparison of Manual vs. Online Grading for Solid Models," in 2013 ASEE Annual Conference \& Exposition, Atlanta, Georgia, 2013, June.

[5] S. J. Kirstukas, "Development and Evaluation of a Computer Program to Assess Student CAD Models," in Proceedings of the 2016 ASEE Annual Conference \& Exposition, New Orleans, LA, 2016.

[6] S. J. Kirstukas and J. Morris, "A Review of File Comparison Utilities for Assessing Student Work," in Proceedings of the 2019 ASEE 73rd Midyear Conference on Engineering Graphics, Berkeley, CA, 2019.

[7] M. Guerci, "Automated Grading System for Engineering Graphics and Computer Aided Design," Rensselaer Polytechnic Institute, Troy, 2003 (not published).

[8] J. Morris, "The Necessity and Results of Autonomous Integrity Evaluation of CAD Files," in Proceedings of the ASEE 2019 73rd Midyear Conference on Engineering Graphics, Berkeley, CA, 2019. 